\title{
NORMAL SUBGROUPS CONTAINED IN THE FRATTINI SUBGROUP ${ }^{1}$
}

\author{
W. MACK HILL ${ }^{2}$ AND CHARLES R. B. WRIGHT
}

\begin{abstract}
Let $H$ be a normal subgroup of the finite group $G$. If $H$ has a subgroup $K$ which is normal in $G$, satisfies $|K|>$ $\left|K \cap Z_{1}(H)\right|=p$ and is not of nilpotence class 2 , then $H$ is not contained in the Frattini subgroup of $G$.
\end{abstract}

All groups considered are finite. The ascending central series of a group $G$ is denoted by $1=Z_{0}(G) \leqq Z_{1}(G) \leqq \cdots$. The Frattini subgroup of $G$, the intersection of all maximal proper subgroups, is denoted by $\Phi(G)$. If $Z_{i}(G)=G$ for some $i, G$ is called nilpotent and the smallest such $i$ is the nilpotence class of $G$, denoted by $\operatorname{cl}(G)$.

E. L. Stitzinger [2] has stated the following result.

THEOREM. Let $H$ be a p-group such that

(i) $\left|Z_{1}(H)\right|=p$;

(ii) there exists an abelian characteristic subgroup $A$ of $H, Z_{1}(H)<A \leqq$ $Z_{2}(H)$.

Then $H$ cannot be a normal subgroup contained in $\Phi(G)$ for any group $G$.

Unfortunately, there is a gap in the proof of Lemma 3 of [2], which is the basis for this theorem. We correct this oversight and prove a generalization of Stitzinger's theorem.

Lemma 3 of [2] says essentially this:

Lemma. Let $A$ be an abelian p-group with subgroup $\langle z\rangle$ of order $p$ such that $A \mid\langle z\rangle$ is elementary abelian. If $T=\{\sigma \in$ Aut $(A) \mid \sigma(z) \in\langle z\rangle\}$ and $S=$ $\left\{\sigma \in T \mid \sigma(z)=z\right.$ and $\sigma(a) a^{-1} \in\langle z\rangle$ for each $\left.a \in A\right\}$, then $S$ is complemented in $T$.

Presented to the Society, January 20, 1972; received by the editors October 12, 1971 and, in revised form, February 3, 1972.

AMS 1970 subject classifications. Primary 20D25; Secondary $20 \mathrm{D} 15$.

Key words and phrases. p-group, normal subgroup, Frattini subgroup.

1 Work supported by National Science Foundation grant GP-29041X.

${ }^{2}$ NSF Science Faculty Fellow, 1970-1971. A portion of the content of this article is contained in this author's doctoral dissertation, written at the University of Cincinnati during 1970-1971 under the direction of Professor Donald Parker. 
Let $x_{1}, x_{2}, \cdots, x_{k} \in A$ such that $\bar{x}_{1}, \bar{x}_{2}, \cdots, \bar{x}_{k}$ is a basis for $A /\langle z\rangle$, where $\bar{x}_{i}=x_{i}\langle z\rangle$. Then each $a \in A$ can be uniquely represented in the form $x_{1}^{a_{1}} \cdots x_{k}^{a_{k} z^{s}}$ with $0 \leqq a_{i}, s<p$. Note that $a_{i}, s$ cannot be taken as elements of the field $F_{p}$ (e.g. take $A=C_{9}$ ). The proof is as follows.

Proof. Let $B=\left\{x_{1}^{a_{1}} \cdots x_{k}^{a_{k}} \mid 0 \leqq a_{i}<p, 1 \leqq i \leqq k\right\}$, and let

$$
M=\{\sigma \in T \mid \sigma(B)=B\} .
$$

$M$ is a subgroup of $T$ and $M \cap S$ is trivial. If $A$ is elementary abelian, then $M=\left\{\sigma \in T \mid \sigma\left(x_{i}\right) \in B, 1 \leqq i \leqq k\right\}$ and Stitzinger's argument is valid, so we assume that $A$ is not of exponent $p$.

Now $M S$ splits over $S$, an abelian (see Lemma 1 of [2]) p-group. By a result of Gaschütz [1, Kapitel I, Hauptsatz 17.4] it suffices to show that $[T: M S]$ is $p$-free.

There is an element $x \in A$ with $|x|=p^{2}$ and, with no loss of generality, $z=x^{p}$. Then $A=\langle x\rangle \times Y$ with $Y$ elementary abelian and we can take $x_{1}=x$ and $\left\langle x_{2}, \cdots, x_{k}\right\rangle=Y$. Let $\pi: A \rightarrow Y$ be the projection homomorphism.

Let $\rho \in T$ be such that $\rho(a) a^{-1} \in\langle z\rangle Y$ for each $a \in A$. Define $\sigma:\{x\} \cup$ $Y \rightarrow A$ by $\sigma(x)=x \pi(\rho(x))$ and $\sigma(y)=\pi(\rho(y))$ for all $y \in Y$. $\sigma$ extends to an element of $T$ and in fact $\sigma \in M$. Since $\rho(a) a^{-1} \in\langle z\rangle Y$ for each $a \in A$, $\sigma(x) \equiv \rho(x) \bmod \langle z\rangle$ and $\sigma(y) \equiv \rho(y) \bmod \langle z\rangle$ for all $y \in Y$. Thus $\sigma^{-1} \rho \in S$ and it follows that $\rho \in M S$. Now $|A|\langle z\rangle Y \mid=p$ and so each $p$-element $\rho$ of $T$ satisfies $\rho(a) a^{-1} \in\langle z\rangle Y$ for all $a \in A$. Hence $M S$ contains all the $p$ elements of $T$ and the Lemma follows.

The arguments of [2], as corrected, are seen to suffice to prove Stitzinger's theorem with the weaker hypothesis that there exists an abelian characteristic subgroup $A$ of $H$ with $A \leqq Z_{2}(H), A \leqq Z_{1}(H)$ and $\left|A \cap Z_{1}(H)\right|=p$. We use this stronger version to prove our main result.

THEOREM. Let $H$ be a p-group with a characteristic subgroup $K$ such that

(i) $\operatorname{cl}(K) \neq 2$;

(ii) $|K|>\left|K \cap Z_{1}(H)\right|=p$.

Then $H$ cannot be a normal subgroup contained in $\Phi(G)$ for any group $G$.

Proof. Let $A=Z_{1}(K) \cap Z_{2}(H)$. $A$ is a nontrivial characteristic subgroup of $H, A \leqq Z_{2}(H)$ and $A \cap Z_{1}(H)=K \cap Z_{1}(H)$. If $A \cap Z_{1}(H)<A$, then the stronger version of Stitzinger's theorem implies the desired result. Assume otherwise, i.e. that $A \leqq Z_{1}(H)$. Then $Z_{1}(K) \leqq Z_{1}(H)$ for, if not, $Z_{1}(K) Z_{1}(H) / Z_{1}(H)$ has nontrivial intersection with $Z_{2}(H) / Z_{1}(H)$. Thus, $\left|Z_{1}(K)\right|=p . K$ is therefore nonabelian and $\operatorname{cl}(K) \geqq 3 . K^{\prime} \cap Z_{2}(K)$ is an abelian characteristic subgroup of $K$, contained in $Z_{2}(K)$, and of order at least $p^{2}$. Hence, by Stitzinger's result, $K$ cannot be a normal subgroup 
contained in $\Phi(G)$ for any group $G$. As $K$ is a characteristic subgroup of $H$, the same is true of $H$.

REMARK. The assumption that the indicated subgroups are characteristic in $H$ can be replaced throughout by the assumption of normality in a fixed extension $G$ of $H(H \triangleleft G)$ with the conclusion that $H \$ \Phi(G)$.

\section{REFERENCES}

1. B. Huppert, Endliche Gruppen. I, Die Grundlehren der math. Wissenschaften, Band 134, Springer-Verlag, Berlin and New York, 1967. MR 37 \#302.

2. E. L. Stitzinger, A nonembedding theorem for finite groups, Proc. Amer. Math. Soc. 25 (1970), 124-126. MR 41 \#3581.

3. — Errata, Proc. Amer. Math. Soc. 34 (1972), 631.

Department of Mathematics, University of Cincinnati, Cincinnati, Ohio 45221

Department of Mathematics, University of Oregon, Eugene, Oregon 97403

Current address (W. Mack Hill): Department of Mathematics, Worcester State College, Worcester, Massachusetts 01602 